\title{
THE PROPOSAL TO “SNAPSHOT” RAIM METHOD FOR GNSS VESSEL RECEIVERS WORKING IN POOR SPACE SEGMENT GEOMETRY
}

\author{
Aleksander Nowak, Ph. D. \\ Gdańsk Univeristy of Technology, Poland
}

\begin{abstract}
Nowadays, we can observe an increase in research on the use of small unmanned autonomous vessel (SUAV) to patrol and guiding critical areas including harbours. The proposal to "snapshot" RAIM (Receiver Autonomous Integrity Monitoring) method for GNSS receivers mounted on SUAV operating in poor space segment geometry is presented in the paper. Existing "snapshot" RAIM methods and algorithms which are used in practical applications have been developed for airborne receivers, thus two main assumptions have been made. The first one is that the geometry of visible satellites is strong. It means that the exclusion of any satellite from the positioning solution don't cause significant deterioration of Dilution of Precision (DOP) coefficients. The second one is that only one outlier could appear in pseudorange measurements. In case of SUAV operating in harbour these two assumptions cannot be accepted. Because of their small dimensions, GNSS antenna is only a few decimetres above sea level and regular ships, buildings and harbour facilities block and reflect satellite signals. Thus, different approach to "snapshot" RAIM is necessary. The proposal to method based on analyses of allowable maximal separation of positioning sub-solutions with using some information from EGNOS messages is described in the paper. Theoretical assumptions and results of numerical experiments are presented.
\end{abstract}

Keywords: GNSS; RAIM; Navigation; Autonomous Vessels

\section{INTRODUCTION}

In 1995 the Full Operational Capability (FOC) of GPS and GLONASS was announced and it started the beginning of a new era in navigation. In spite of GLONASS lost his FOC after a few years, the field of satellite navigation applications continued to grow. In 2011 GLONASS regained its FOC and now together with GPS they are foundation of GNSS (Global Navigation Satellite System). The simplicity of obtaining position coordinates and integration with other devices and navigation systems has caused that nowadays GNSS is the main way of determining the location of objects in the various applications [1][2]. However, GNSS positioning in not free from the constrains. The two main ones are: limiting the number of observed satellites caused by terrain obstacles both natural and those which are result of human activities (e.g. buildings) and integrity, understood as the ability of the system to alert the users in a given amount of time that the fix errors exceeded the permissible limit. GNSS receivers have become the main source of information about the location of autonomous unmanned vehicles and in such applications high level of integrity is as much important as fixes accuracy [3].

Integrity was one of the main problem considered in phase of work on the system GPS, which has resulted in many research papers, but the main area of concern was air navigation and the focus was on the development of methods to assess the correctness of GPS fixes during the flight [4]. Two main solutions have been proposed and applied in practice: external methods (integrity monitoring by the use of external infrastructures) and internal (integrity monitoring directly by the receiver). The second ones are called RAIM (Receiver Autonomous Integrity Monitoring). Among them, a separate group are those that assess the correctness of the coordinates on the basis of a single measurement of pseudoranges for at least 5 satellites - they are called "snapshot" RAIM [5]. Both external and internal methods solve the problem of integrity in the open air in a satisfactory way. However, if the outlier in pseudorange measurement is not caused by improper functioning of the satellite but by obstacles which reflect satellite signals these methods cannot be used. External methods are not able to detect such errors because of fault detection process is carried out by the monitoring station and do not take into account the local conditions at the place where the user is (see Fig. 1). On the other hand, internal methods were designed for the air navigation and they are based on two main assumptions. The first one is that only one outlier can appear in pseudorange measurements. It was adopted on the basis of high reliability of the satellites, which causes that faulty work of two satellites at the same 
time is unlikely. The second one is related to the method used to integrity monitoring. It is that the exclusion of any satellite from the positioning solution don't cause significant deterioration of Dilution of Precision (DOP) coefficients. As far as we talk about navigation in the open air there are no problems associated with these assumptions. But in case of SUAV (Small Unmanned Autonomous Vessel) operating in the harbour such approach cannot be accepted. Because of their small dimensions, GNSS antenna is only a few decimetres above sea level and regular ships, buildings and harbour facilities block and reflect satellite signals. On the one hand it causes poor space segment geometry (it means that exclusion of one satellite from the positioning solution causes significant deterioration of DOP) and on the other one more than one outlier can appear in pseudorange measurements (see Fig. 1). In this paper proposal of different approach is described.

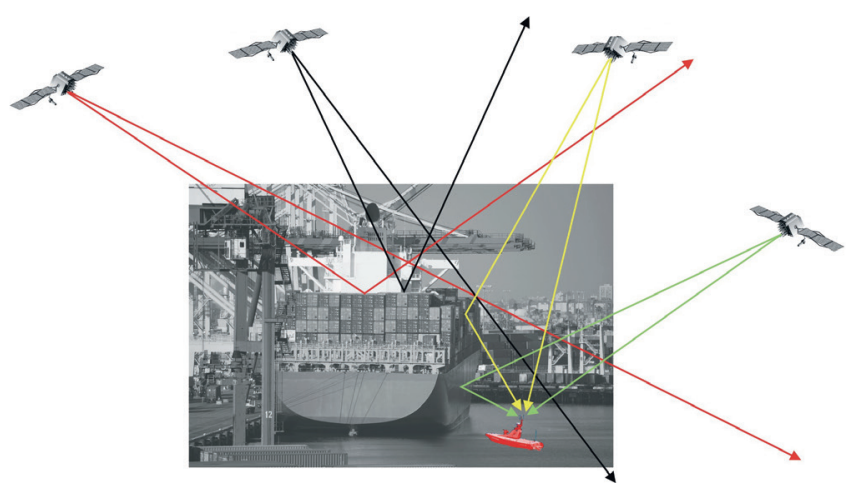

Fig. 1. Possible sources of outliers in pseudorange measurements in case of SUAV operating in the harbour

\section{THE IDEA OF THE PROPOSED RAIM METHOD}

The idea of the proposed RAIM method has been already described in [6]. It is deep modification of Maximum Solutions Separation (MSS) method proposed by R.G. Brown and McBurney in 1987 and described in [7]. The idea of the MSS method is based on the study of the maximum separation between positioning sub-solutions. The number of sub-solutions is equal the number of observed satellites, and they are formed by successive exclusion of one satellite from the positioning solution. If maximum separation of sub-solutions is greater than the acceptable one it means that there is outlier in pseudorange measurements. Described idea is shown in Fig. 2 and Fig. 3.

In Fig. 3 the idea of fault identification is also illustrated. Because of after exclusion of SV 1 from positioning solution, sub-solution (SVs: 2,3,4,5) was within acceptable limits it means that in measurement to SV 1 outlier appeared.

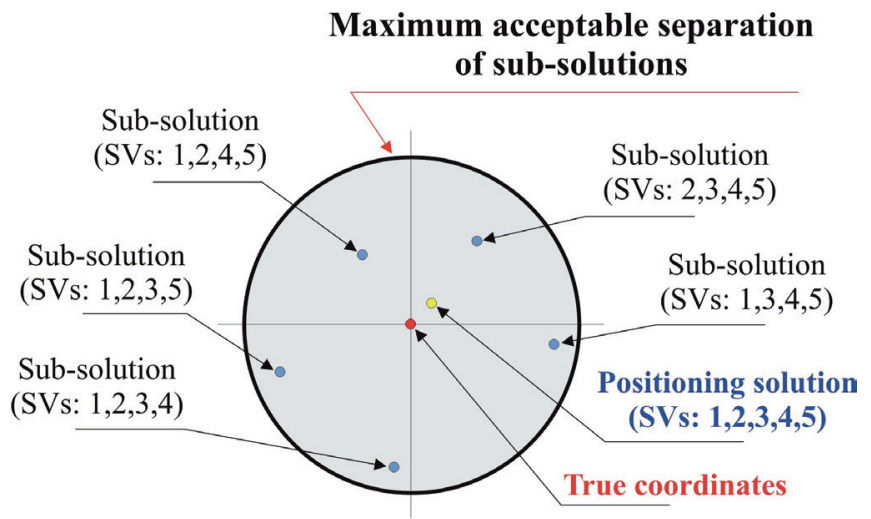

Fig. 2. Sub-solutions separation if there are no outliers in pseudorange measurements

Maximum acceptable separation of sub-solutions

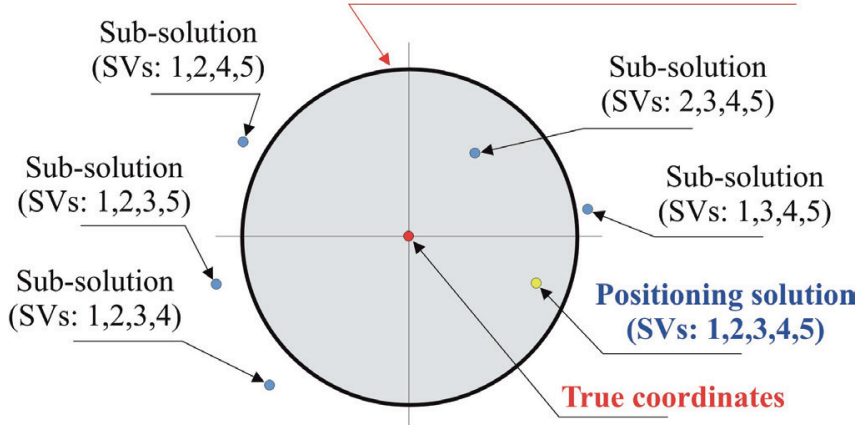

Fig 3. Sub-solutions separation if there is outlier in pseudorange measurement to $S V 1$

The MSS RAIM method has not found practical application due to the inability to precisely determine maximum acceptable separation of sub-solutions. In particular it was impossible in case of poor space segment geometry, because observed separation of sub-solutions could be the result of both: outlier in pseudorange measurements or significant deterioration of DOP as a result of the elimination one of the satellites from the positioning solution. This problem is described, inter alia, in $[7,8]$. The method proposed by author is an attempt to eliminate the above disadvantage. The research conduce on influence of pseudorange measurement errors and space segment geometry on GPS fixes distribution described, inter alia, in $[9,10,11,12,14, .15,16]$ have shown that the extended analysis of the spatial distribution of the positioning sub-solutions can significantly improve the protection level of the existing RAIM "snapshot" methods.

The study shows that, despite the fact that at the poor space segment geometry separation of sub-solutions is similar in both cases: the outliers appeared in pseudorange measurements or they didn't appear, their spatial distribution is different. Thus, if we have the pattern of spatial distribution of sub-solutions it could be possible to determine whether there are outliers in pseudorange measurements or not, by comparing the actual distribution to the pattern. Similarity will mean that pseudorange measurements contain only random errors. The pattern of spatial distribution of 
sub-solutions in case of outlies absence will be constructed in real time, on the basis of the corrections transmitted from the Satellite Based Augmentation System (SBAS) - in Europe from EGNOS.

The simplified algorithm of proposed fault detection method is as follows:

- $\quad$ estimating current random errors of pseudorange measurements on the basis of data received from the geostationary satellites of SBAS system,

- building the pattern of spatial distribution of sub-solutions,

- calculating of real spatial distribution of sub-solutions,

- comparing the pattern to real distribution. If both are similar it means that there are no outliers in pseudorange measurements. If not - outliers appeared.

\section{THE SPATIAL DISTRIBUTION OF SUB- SOLUTIONS IN MSS METHOD}

In practical applications we are interested in the spatial distribution of sub-solutions in relation to position calculated by the receiver, instead of to the true coordinates, because we do not know them. It is additional complication which caused that the MSS method has not been applied in practice. The research on spatial distribution of sub-solutions that were done using the software for simulating GNSS measurements, allowed to examine the issue more precisely. The results of numerical experiments were described between others in [9]. The conclusions of the study can be summarized as follows:

- $\quad$ if there are no outliers in pseudorange measurements, the distribution of sub-solutions in relation to positioning solution (coordinates computed on the base of observations done to all visible satellites) is a set of line segments intersecting in the middle of its length (number of line segments is equal to the number of visible satellites) - see Fig. 4 .,

- $\quad$ if there are outliers in pseudorange measurements, the distribution of sub-solutions in relation to positioning solution is a set of line segments, which lengths are the same as in the absence of outliers but they do not intersect - see Fig. 5 and 6.

To illustrate the above, an example of the numerical simulation results is described below.

\section{AN EXAMPLE OF THE NUMERICAL SIMULATION RESULTS}

Using software simulator of GNSS measurements (described in [15]) geometrical layout of 5 GPS satellites was drawn. Azimuths and elevations of the satellites are presented in Table I. Then simulation of 86400 fixes was done, wherein it was assumed that errors of pseudorange measurements were modelled as independent random variables with $\mathrm{N}[0,1]$ distribution and standard deviation equal $6 \mathrm{~m}$. In the first step of the simulation there were no outliers in measurements and in the second one the outlier equal $200 \mathrm{~m}$ and then $100 \mathrm{~m}$ appeared in pseudorange measurement to SV1. Obtained sub-solutions distribution related to the position computed on the base of all 5 observed satellites are presented in Fig. 4,5 and 6.

Tab.1. Azimuths and elevations of the drawn satellites during simulation

\begin{tabular}{|c|c|c|}
\hline SV & Azimuth [1] & Elevation [Y] \\
\hline 1 & 328,1 & 53,7 \\
\hline 2 & 309,3 & 05,8 \\
\hline 3 & 011,2 & 34,7 \\
\hline 4 & 052,5 & 82,3 \\
\hline 5 & 240,6 & 44,2 \\
\hline
\end{tabular}

Sub-solution (SVs: 2,3,4,5)

Sub-solution (SVs: 1,3,4,5)

Sub-solution (SVs: $1,2,4,5)$

Sub-solution

(SVs: 1,2,3,5)

Sub-solution (SVs: $1,2,3,4)$

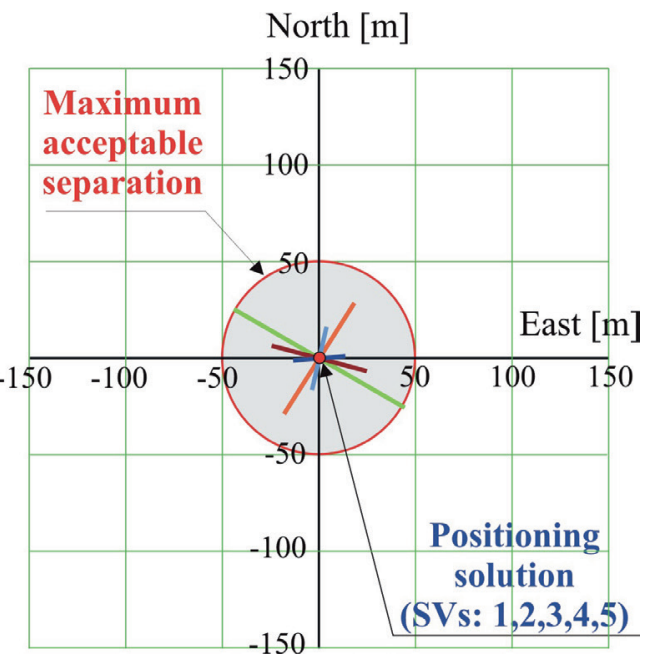

Fig. 4. Obtained sub-solutions distribution related to the positioning solution if there are no outliers in pseudorange measurements

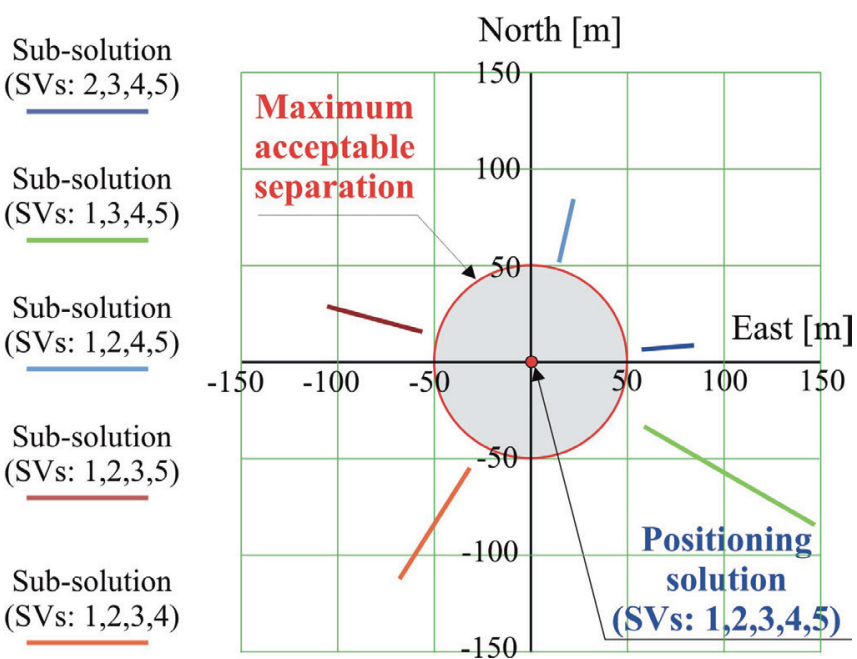

Fig. 5. Obtained sub-solutions distribution related to the positioning solution if outlier equal $200 \mathrm{~m}$ appeared in pseudorange measurement to SV1 




Fig. 6. Obtained sub-solutions distribution related to the positioning solution if outlier equal $100 \mathrm{~m}$ appeared in pseudorange measurements to SV1

The Fig. 4, 5 and 6 show, that determination of maximum acceptable separation of sub-solutions as a circle (or as a sphere in 3D) on the basis of an assumption concerning standard deviation of measurement random errors $\left(\boldsymbol{\sigma}_{\mathrm{p}}\right)$ is not very efficient. It can be noticed in Fig. 4 that maximum acceptable separation resulting from the deployment of satellites and $\sigma_{p}$ is equal $50 \mathrm{~m}$. If outlier appears in pseudorange measurement to SV1 its value must be at least $180 \mathrm{~m}$ to get a $100 \%$ probability of detection. Otherwise the calculated separation of sub-solutions may be in the range of maximum acceptable separation (compare Fig. 5 and 6) which will result in outliers misdetection. On the other hand, if the value of maximum acceptable separation will be reduced, false alarms will appear, due to possibility of exceeding separation limit although there are no outliers in pseudorange measurements. It is typical situation in case of poor space segment geometry, described, inter alia, in $[9,10,11,12]$ and main cause of very low protection level when number of visible satellites is lower than 7 .

Thus, it seems to be clear that more sophisticated analyse is necessary. The presented proposal to modification of MSS method is based on estimation of current random errors of measurements on the basis of data received from the geostationary satellites of SBAS system. They allow to compute corrections to pseudorange measurements and in proposed RAIM method it is assumed that they are equal current random errors with the opposite sign. Of course it is not quite true, because of SBAS corrections don't eliminate the entire size of the random errors but it is expected that such approximation allows to improve the level of outliers detection. Of course as a source of information about current random errors of measurements DGPS system could be also used but DGPS receivers are bigger, heavier and more expensive than EGNOS ones (additional MSK module is necessary) so their application in SUAVs is not so convenient.

\section{THE SPATIAL DISTRIBUTION OF SUB- SOLUTIONS IN MODIFIED MSS METHOD}

Below an example of simulation results is described. Simulation concerned the same deployment of the satellites as described in previous section. This time drawn values of pseudorange measurements random errors were as follow: SV1: -3,2 m, SV2: 7,9 m, SV3: -5,4 m, SV4: -2,6 m, SV5: 6,1 m. Drawn values of measurements errors have given distribution of sub-solutions as shown in Fig. 7

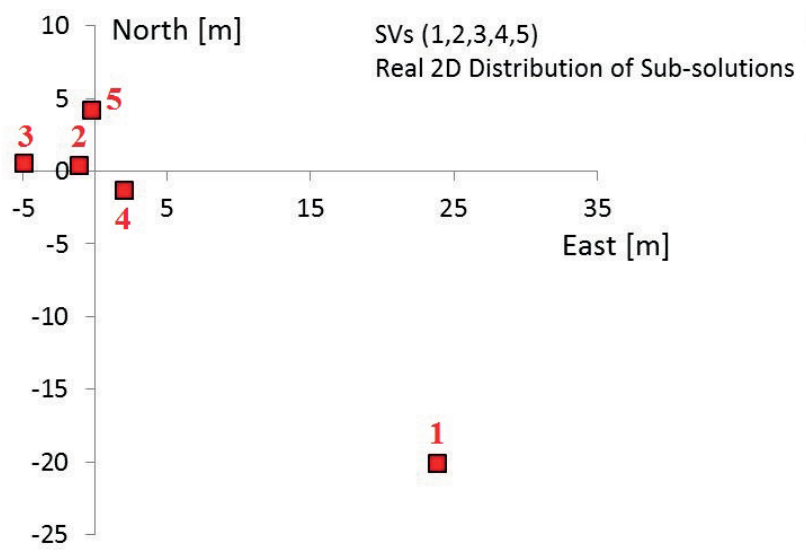

Fig. 7. Distribution of sub-solutions for drawn values of pseudorange measurements random errors. Red squares marked shift of sub-solutions related to the positioning solution. Numbers at squares indicate number of satellite excluded from the positioning solution

Because in reality random errors are unknown, in proposed method they are estimated on the base of SBAS message. It was assumed, that SBAS corrections eliminate about $85 \%$ of random measurements errors, therefore possible corrections were randomly generated. Then was assumed, that random measurements errors are equal to the corrections with the opposite sign and as a result the following estimators of random measurements errors were obtained: SV1: $-2,9 \mathrm{~m}$, SV2: 5,9 m, SV3: -4,5 m, SV4: -2,4 m, SV5: 5,3 m.

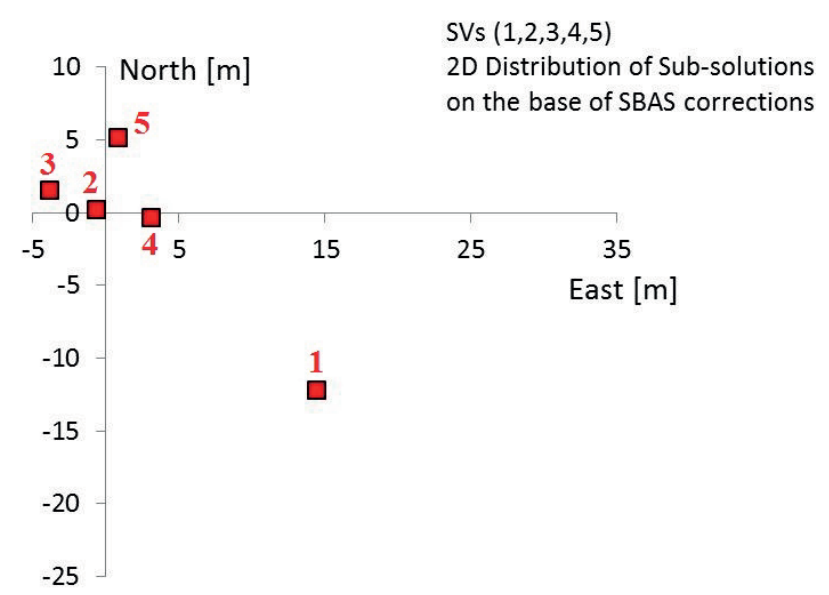

Fig. 8. Distribution of sub-solutions computed on the base of information from the SBAS message. Red squares marked shift of sub-solutions related to the positioning solution. Numbers at squares indicate number of satellite excluded from the positioning solution 
Distribution of sub-solutions computed on the base of simulated information from SBAS message is presented in Fig. 8. Fig. from 9 to 13 shows distributions in case of outliers presence.

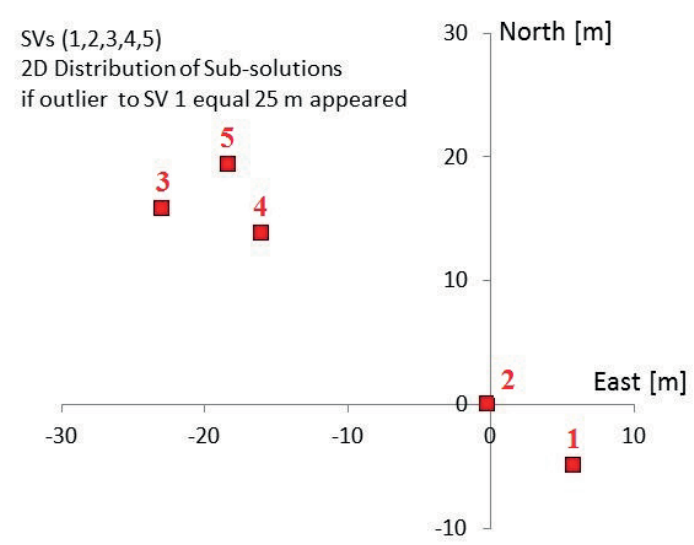

Fig. 9. Distribution of sub-solutions if outlier equal $25 \mathrm{~m}$ appeared in pseudorange measurement to SV1. Red squares marked shift of sub-solutions related to the positioning solution. Numbers at squares indicate number of satellite excluded from the positioning solution

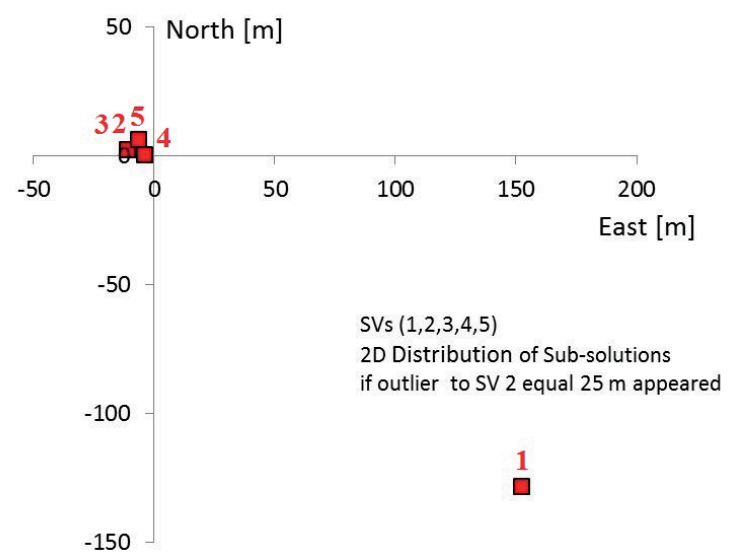

Fig. 10. Distribution of sub-solutions if outlier equal $25 \mathrm{~m}$ appeared in pseudorange measurement to SV2. Red squares marked shift of subsolutions related to the positioning solution. Numbers at squares indicate number of satellite excluded

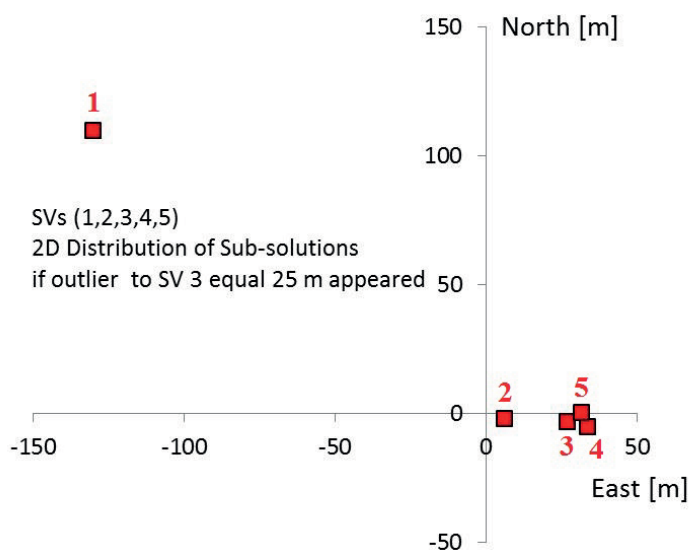

Fig. 11. Distribution of sub-solutions if outlier equal $25 \mathrm{~m}$ appeared in pseudorange measurement to SV3. Red squares marked shift of sub-solutions related to the positioning solution. Numbers at squares indicate number of satellite excluded from the positioning solution

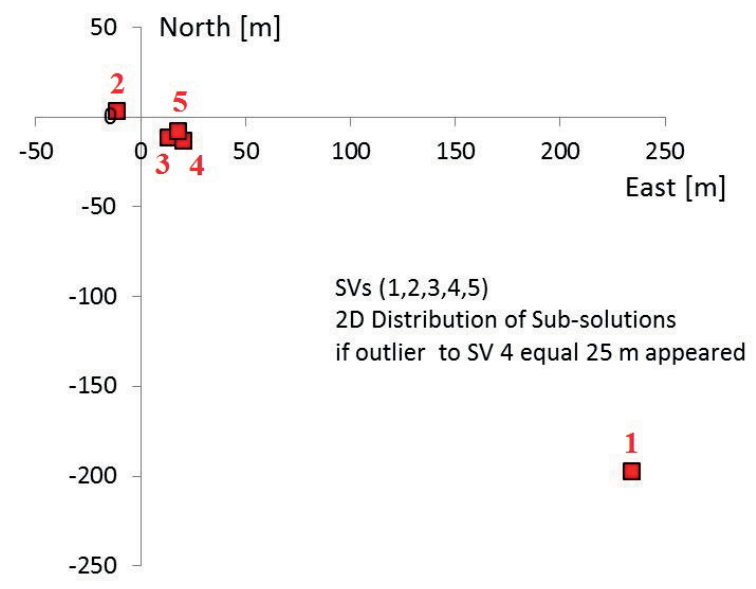

Fig. 12. Distribution of sub-solutions if outlier equal $25 \mathrm{~m}$ appeared in pseudorange measurement to SV4. Red squares marked shift of sub-solutions related to the positioning solution. Numbers at squares indicate number of satellite excluded from the positioning solution

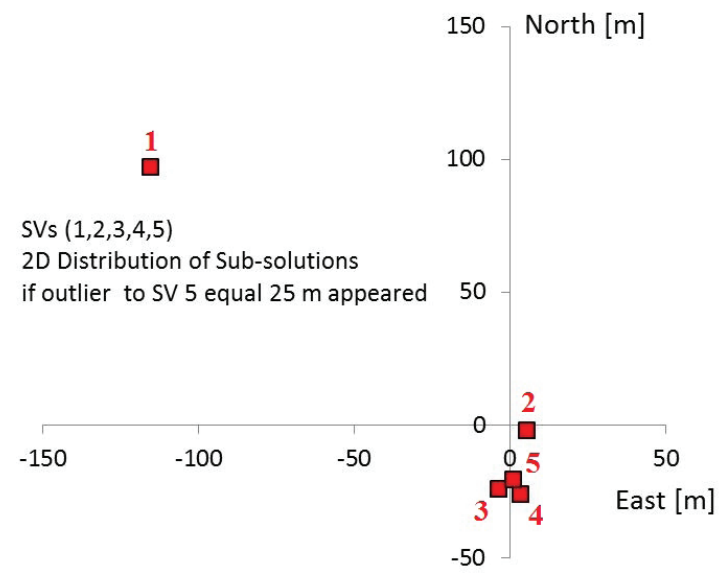

Fig. 13. Distribution of sub-solutions if outlier equal $25 \mathrm{~m}$ appeared in pseudorange measurement to SV5. Red squares marked shift of sub-solutions related to the positioning solution. Numbers at squares indicate number of satellite excluded from the positioning solution

It can be easily noticed, that if outlier appeared in measurements to SVs: 2, 3, 4, 5 classic MSS method is able to detect it, because separations of sub-solutions were bigger than maximum acceptable separation computed on the base of assumed standard deviation of random errors and layout of the satellites (in presented example $50 \mathrm{~m}$ ). But in case of outlier in measurement to SV1, separation of sub-solutions is almost half less than acceptable one (is $27 \mathrm{~m}$ ). It can be also observe if we compare Fig. 7 and 9. In such situation classic MSS method misses outlier. But if we compare spatial distribution of sub-solutions, they differ depending on outlier appeared or not (see Fig. 7, 8 and 9). Thus it seems, that extended analyse of distribution of sub-solutions can improve protection level of proposed RAIM method in comparison with the classic MSS one. 


\section{SUMMARY}

The presented examples of the numerical experiment results seem to confirm hypothesis that with the pattern of spatial distribution of sub-solutions it is possible to improve protection level of classic "snapshot" RAIM method. The pattern can be built based on differential corrections received from SBAS system. Of course further research are necessary to confirm preliminary results. Thus, proposed algorithm will be implemented in GNSS measurements simulator and extensive tests will be done. They will be focused on determination of protection level with given probability of misdetection and false alarm. This will allow to reliably determine the effectiveness of the proposed solution compared to the current ones.

\section{REFERENCE}

1. A. Janowski, A. Jurkowska, D. Lewczuk, J. Szulwic, A. Zaradny, Assessment of cliff stability after the demolition of the engineering facilities, 14th SGEM GeoConference on Science and Technologies in Geology, Exploration and Mining, ISBN 978-619-7105-08-7 ISSN 1314-2704, 2 (2014) 115-124, DOI: 10.5593/SGEM2014/B12/S2.016

2. J. Dąbrowski, M. Kulawiak, M. Moszyński, K. Bruniecki, Ł. Kamiński, A. Chybicki, A. Stepnowski, Real-time web-based GIS for analysis, visualization and integration of marine environment data. Information Fusion and Geographic Information Systems, Lecture Notes in Geoinformation and Cartography, (2009) 277-288, DOI: 10.1007/978-3-642-00304-2_19

3. B.W. Parkinson, P. Axelrad: Autonomous Integrity Monitoring Using the Pseudorange Residuals, NAVIGATION 35/2 (1988) 255-274

4. C.L. Young: New Concept of Independent GPS Integrity Monitoring, NAVIGATION 35/4 (1988) 483-501

5. R.G. Brown: Receiver Autonomous Integrity Monitoring, Global Positioning System: Theory and Applications vol. II, Denver, 1996, pp.143-164

6. A. Nowak: Nowa metoda oceny dokładności wyznaczeń GNSS na potrzeby monitoringu pojazdów, LOGISTYKA 3 (2014) 4706-4717

7. R.G. Brown, P.W. McBurney: Self-Contained GPS Integrity Check Using Maximum Solution Separation as the Test Statistic, Proceedings of the International Technical Meeting of The Institute of Navigation (1987) 263-268

8. M.A. Sturza: Fault Detection and Isolation (FDI) Techniques for Guidance and Control Systems, AGARDOGRAPH 314 (1991), AGARD, NATO
9. A. Nowak: Influence of Pseudorange Measurement Errors and Space Segment Geometry on GPS Fixes Distribution, Advances in Computer Science-Network Centric Warfare, Gdynia, 2009, pp.148-154

10. A. Nowak: Protection Level of "Snapshot" RAIM Methods in Poor Geometry of Satellites, Advances in Computer ScienceNetwork Centric Warfare, Gdynia, 2009, pp.156-162.

11. C. Specht, A. Nowak: Limitations of Satellite Positioning in Inland Shipping, Polish Journal of Environmental Studies 16, No 6B (2007) 83-86

12. A. Nowak, D. Szulc: Poziom Ochrony metod RAIM typu "snapshot" w warunkach niekorzystnej geometrii segmentu kosmicznego, LOGISTYKA 6 (2009)

13. A. Nowak: Problemy pozycjonowania satelitarnego w aglomeracjach miejskich, LOGISTYKA 3 (2011)

14. A. Nowak: Symulacje numeryczne wpływu przeszkód terenowych na dokładność wyznaczeń GNSS, LOGISTYKA 3 (2011)

15. A. Janowski, A. Nowak, M. Przyborski, J. Szulwic: Mobile indicators in GIS and GPS positioning accuracy in cities, Rough Sets and Intelligent Systems Paradigms, Lecture Notes in Computer Science 8537 (2014) 309-318

16. A. Nowak, C. Specht: Computer Simulator of GNSS Measurements, Annual of Navigation 12 (2008)

\section{CONTACT WITH AUTHOR}

\author{
Aleksander Nowak \\ Gdansk University of Technology \\ G. Narutowicza 11/12 St. \\ 80-233 Gdansk \\ Poland \\ e-mail: aleksander.nowak@geodezja.pl \\ phone: +48 883911654
}

